\title{
8 Entropy Production of Atmospheric Heat Transport
}

\author{
Takamitsu Ito ${ }^{1}$ and Axel Kleidon ${ }^{2}$ \\ ${ }^{1}$ Program in Atmospheres, Oceans and Climate, Massachusetts Institute of Tech- \\ nology, Cambridge, MA 02139, USA; \\ ${ }^{2}$ Department of Geography, 2181 Lefrak Hall, University of Maryland, College \\ Park, MD 20742, USA.
}

\begin{abstract}
We examine the rate of entropy produced by the atmospheric general circulation and the hypothesis that it adjusts itself towards a macroscopic state of maximum entropy production. First, we briefly review thermodynamics of a zonally-averaged, dry atmosphere. We examine the entropy balance of a dry atmosphere, and identify the key processes that lead to entropy production. Frictional dissipation and diabatic eddy transfer are the major sources of entropy production, and both processes are dominated by baroclinic eddies in the middle latitudes. Secondly, we derive a simple solution for the upper bound of entropy production from the energy balance constraint, which can be compared to the simulated temperature distribution simulated by an idealized GCM. These temperatures agree well with the MEP solution in the mid-latitude troposphere. However, there are significant differences in tropics where the Hadley circulation controls the large-scale temperature distribution. Finally, we show that the simulated entropy production is sensitive to model resolution and the intensity of boundary layer friction, and explore the significance of dynamical constraints. We close with a discussion of the implications of the MEP state for global climatology.
\end{abstract}

\subsection{Introduction}

The atmospheric circulation is driven by the temperature gradient $\Delta T_{E, P}$ between the equatorial and polar regions as a result of differences in solar irradiation. This temperature gradient is not fixed, but is affected by the amount of heat transport associated with the atmospheric circulation. The transport of heat from the warmer tropics to the colder poles leads to entropy production. Paltridge (1975) first suggested that the atmospheric circulation adjusts itself to a macroscopic state of maximum entropy production (MEP). Several authors applied the MEP hypothesis to energy balance climate models, for example, Paltridge (1978), Nicolis et al. (1980), Grassl (1981), and Pujol et al. (2000). They suggest that the MEP solutions are in plausible agreement with observed variables characterizing the zonal mean present-day climate. Lorenz et al. (2001) suggests that the MEP also applies to the atmospheres of other planets such as Mars and Titan (see also Lo- 
renz, this volume). While empirical support for the MEP hypothesis has been accumulating, the fundamental mechanisms are not yet fully understood.

Lorenz (1960) suggested that the atmosphere maximizes the production rate of available potential energy (APE), which is essentially equivalent to the MEP hypothesis of Paltridge when appropriate definition of the entropy production is considered (Ozawa et al. 2003). Lorenz's hypothesis of the maximum APE production considers the general circulation of the atmosphere as a heat engine of maximum efficiency in which the production of mechanical work is maximized for given solar forcing. In a statistical steady state, the production rate of APE must balance the rate of dissipation. Ozawa et al. (2003) derive a simple linear relationship between the production rate of APE and the entropy production due to the turbulent dissipation.

Recently, Dewar (2003) studied the theoretical basis for the MEP hypothesis based on the statistical mechanics of open, non-equilibrium systems. The state of MEP emerges as the statistical behavior of the macroscopic state when the information entropy is maximized subject to the imposed constraints. Dewar's theory is generally applicable to a broad class of the steady state, non-equilibrium system, such as fluid turbulence. Studies of Paltridge and others could be considered as a particular representation of the MEP principle in the climate system.

The MEP hypothesis has been applied to different types of climate models with various assumptions. Shutts (1981) applied the MEP hypothesis to the two-layer quasi-geostrophic model, and maximized the entropy production with the constraints of energy and enstrophy conservation. The extremal solution of Shutts is somewhat comparable to the ocean gyre circulation. Ozawa and Ohmura (1997) applied the MEP hypothesis to radiative convective equilibrium model, and reproduced a reasonable vertical temperature profile associated with the MEP state. Shimokawa and Ozawa (2002, also this volume) examined the entropy production in the multiple steady states of an ocean general circulation model, and suggest that the system tends to be more stable at higher rates of entropy production. Kleidon (2004) showed with a simple two box model of the surface-atmosphere system that the partitioning of energy at the Earth's surface into radiative and turbulent fluxes can also be understood by MEP. The MEP hypothesis was also recently demonstrated to emerge from atmospheric General Circulation Model (GCM) simulations in which model resolution and boundary layer friction was modified (Kleidon et al. 2003). These results may also serve as empirical supports for the basic concept of the MEP in the climate system.

This chapter investigates the physical processes that control entropy production in the atmospheric general circulation and how they may be related to the MEP hypothesis. In particular, we use atmospheric GCMs to simulate large-scale eddies in the atmosphere and examine their role in setting the atmospheric heat transport and the associated entropy production. We first review the entropy balance in the zonally-averaged dry atmosphere and consider the entropy balance in the model. Secondly, we analytically derive the upper bound of entropy production, and examine how close the simulated entropy production is to the theoretical upper bound. We also compare the temperature distribution of the analytic MEP solution to that of the numerical simulation and discuss the importance of dynami- 
cal constraints, imposed by angular momentum conservation. We then show that simulated entropy production is sensitive to model resolution and the intensity of boundary layer friction, and shows a characteristic maximum. We close with a discussion of the implications of the MEP state for climatology.

\subsection{Entropy production in an idealized dry atmosphere}

We briefly review the thermodynamic balance of a zonally-averaged dry atmosphere. A change in specific entropy, $d s$, of an air parcel with temperature $T$ is defined as $T d s=d Q$. A detailed derivation of atmospheric thermodynamics can for example be found in Gill (1982). Following the trajectory of an air parcel, the change in the specific entropy is related to the rate of diabatic heating, $D Q / D T$ :

$$
\frac{D s}{D t}=\frac{1}{T} \frac{D Q}{D t}
$$

The potential temperature $\theta$ of the air parcel can be defined in terms of its specific entropy, $s=c_{p} \ln \theta$, where $c_{p}$ is specific heat of dry air at constant pressure. The thermodynamic equation can then be derived from eqn (8.1):

$$
\frac{D \theta}{D t}=\frac{1}{c_{p}} \frac{\theta}{T} \frac{D Q}{D t}
$$

Adiabatic processes conserve both specific entropy and potential temperature. For a dry atmosphere, the diabatic heating term includes heating due to thermal diffusion, viscous dissipation, and radiative fluxes. We parameterize the radiative heating and the frictional dissipation as in Held and Suarez (1994) (hereafter, HS94), which is often used to evaluate the hydrodynamics of atmospheric general circulation and climate models. The radiative transfer is parameterized as a Newtonian damping of local temperature to the prescribed radiative-convective equilibrium profile $T_{e q}$ :

$$
\frac{D Q}{D T}=-c_{p} k_{T}\left(T-T_{e q}\right)+k_{U}\left(u^{2}+v^{2}\right)
$$

The second term on r.h.s. represents the heating due to viscous dissipation, which is often neglected since its magnitude is very small (a few percent) compared to that of the radiative heating. Here, we include this term for consistency in the energy balance. The frictional damping coefficient $k_{U}$, the radiative cooling coefficient $k_{T}$, and the radiative-convective equilibrium temperature profile $T_{e q}$, are prescribed functions of latitude and pressure. For the detailed distribution of $T_{e q}$, $k_{T}$ and $k_{U}$, see HS94.

Next, we zonally average the thermodynamic equation (8.2). It is convenient to define the Exner function $\pi=\left(p / p_{0}\right)^{\kappa}$, where $p_{0}$ is the surface pressure and $\kappa=2 / 7$. With this definition, we can express the thermodynamic equation as 


$$
\frac{\partial \bar{\theta}}{\partial t}+\nabla \cdot \bar{u} \bar{\theta}=-\nabla \cdot \overline{u^{\prime} \theta^{\prime}}+\frac{1}{c_{p} \pi(p)} \frac{\overline{D Q}}{D t}
$$

Here, zonally averaged quantities are overlined such as $\bar{A}$, and the deviations from the mean are written as $A^{\prime}=A-\bar{A}$. We obtain the zonal mean entropy balance equation by multiplying both sides of eqn. (8.4) by $c_{p} / \bar{\theta}$ :

$$
\frac{\partial \bar{s}}{\partial t}+\nabla \cdot \overline{u s}=-c_{p} \nabla \cdot\left(\frac{\overline{u^{\prime} \theta^{\prime}}}{\bar{\theta}}\right)-c_{p} \frac{\overline{u^{\prime} \theta^{\prime}} \cdot \nabla \bar{\theta}}{\bar{\theta}^{2}}+\frac{1}{T} \frac{\overline{D Q}}{D t}
$$

The r.h.s. of this equation contains two components of eddy fluxes that contribute to entropy production. The first term on the r.h.s. represents the adiabatic component of the eddy transfer which vanishes when integrated globally. The second term is the diabatic component of the eddy transfer which does not vanish when integrated globally.

\subsubsection{Global budget of energy and entropy}

Globally integrated, the radiative heating must be zero for a steady state. Thus, we have

$$
<c_{p} k_{T}\left(T-T_{e q}\right)>=0
$$

with the brackets denoting the global integral, $<>=-\int d x d y d p / g$. Combining eqs.(8.3) and (8.5) and integrating globally, we derive the global entropy balance:

$$
<\sigma_{T O T}>=\left\langle\frac{c_{p} k_{T}\left(\bar{T}-T_{e q}\right)}{\bar{T}}\right\rangle=\left\langle-c_{p} \frac{\overline{u^{\prime} \theta^{\prime}} \cdot \nabla \bar{\theta}}{\bar{\theta}^{2}}+\frac{k_{U}\left(\overline{u^{2}+v^{2}}\right)}{T}\right\rangle
$$

The globally integrated entropy production $\left\langle\sigma_{T O T}\right\rangle$ can be expressed in terms of the net outgoing entropy flux, and it is balanced by the integral of local entropy production through diabatic eddy fluxes and frictional dissipation. This particular formulation does not involve entropy production due to radiative transfer, dry and moist convection and other moist processes (see e.g. Pauluis, this volume). For example, eqn. (8.7) implies that $\left\langle\sigma_{T O T}\right\rangle=0$ when the atmosphere is in radiativeconvective equilibrium $T=T_{e q}$. This definition of entropy production is essentially identical to the definition of Paltridge (1975).

\subsubsection{Sources of entropy production}

The atmospheric general circulation has internal sources of entropy due to dissipative processes. We diagnose the simulated fields, quantify the spatial distribution of these entropy sources, and illustrate the dynamical process controlling the entropy production. Frictional dissipation and diabatic eddy fluxes can be diag- 
nosed directly from the simulated fields. We can define a local entropy production, which includes a frictional component $\sigma_{\text {fric }}$ and an eddy component $\sigma_{\text {eddy }}$ :

$$
\begin{gathered}
\sigma_{\text {fric }}=\frac{k_{U}\left(\bar{u}^{2}+\bar{v}^{2}+\overline{u^{\prime 2}}+\overline{v^{\prime 2}}\right)}{\bar{T}} \\
\sigma_{e d d y}=-c_{p} \frac{\overline{u^{\prime} \theta^{\prime}} \cdot \nabla \bar{\theta}}{\bar{\theta}^{2}}
\end{gathered}
$$

The units of $\sigma_{\text {fric }}$ and $\sigma_{e d d y}$ are $\mathrm{W} \mathrm{K}^{-1} \mathrm{~kg}^{-1}$, and they satisfy $\sigma_{T O T}=\sigma_{\text {fric }}+\sigma_{\text {eddy }}$ (see eqn. 8.7). In the following, we discuss entropy production rates per unit area, that is, integrated over the vertical column of air and in units of $\mathrm{mW} \mathrm{m}^{-2} \mathrm{~K}^{-1}$, and not per unit weight.

\subsubsection{Theoretical upper bound of entropy production}

In order to derive an upper bound of the global rate of entropy production $\sigma_{T O T}$, as described by eqn. (8.7), we maximize $\sigma_{T O T}$ subject to the constraint of global energy balance, as described by eqn. (8.6). Dynamical constraints, as for instance imposed by the conservation of angular momentum, are not included in the constraint, so the model can be considered to be an energy balance model. We introduce a Lagrange multiplier $\mu$ and combine the constraint eqn. (8.6) and the cost function $\left\langle\sigma_{T O T}>\right.$ :

$$
<\sigma_{T O T}>=\left\langle\frac{c_{p} k_{T}\left(\bar{T}-T_{e q}\right)}{\bar{T}}\right\rangle+\mu\left\langle c_{p} k_{T}\left(\bar{T}-T_{e q}\right)\right\rangle
$$

The rate of entropy production $\left\langle\sigma_{T O T}\right\rangle$ is then extremized by setting $\delta<\sigma_{T O T}>=0$. The resulting Euler-Lagrange equation for this extremization is:

$$
T_{M E P}=\mu^{-1 / 2} T_{e q}^{1 / 2}
$$

$T_{M E P}$ is the temperature distribution associated with the upper bound of entropy production. The Lagrange multiplier $\mu$ is calculated by combining eqn. (8.6) and (8.11):

$$
\mu^{-1 / 2}=<k_{T} T_{e}>/<k_{T} T_{e q}^{1 / 2}>
$$

The maximum in entropy production is then calculated by using eqn.(8.10):

$$
<\sigma_{\mathrm{TOT}, \mathrm{MEP}}>=<c_{p} k_{T}\left(1-\mu^{1 / 2} T_{e q}{ }^{1 / 2}\right)>
$$

Applying definitions of $k_{T}$ and $T_{e q}$ following HS94, we find $\sigma_{\mathrm{TOT}} \approx 8.4 \mathrm{~mW} \mathrm{~m}^{-2}$ $\mathrm{K}^{-1}$. The resulting temperature profile $T_{M E P}$ is shown in Fig. 8.1. 

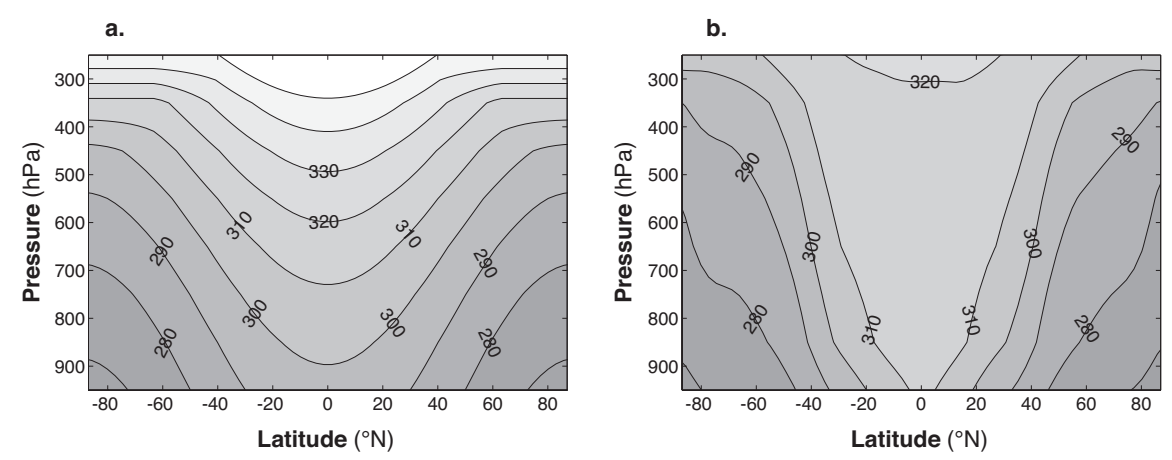

Fig. 8.1. Distribution of zonally-averaged potential temperature (a) resulting from a state of maximum entropy production derived analytically and (b) simulated with a GCM.

\subsection{Testing maximum entropy production with atmospheric general circulation models}

The analytic form of the MEP solution given by eqn. (8.13) and the associated temperature distribution given by eqn. (8.11) is compared with the simulated properties of an atmospheric GCM. We first present the simulated entropy production of an atmospheric general circulation model including its latitudinal variation. Next, we compare the simulated temperatures to those associated with the theoretical upper bound of entropy production. In the third part we then discuss the sensitivity of simulated entropy production to model resolution and boundary layer turbulence in order to illustrate the conditions for MEP states associated with the atmospheric circulation.

\subsubsection{Simulated entropy production in the climatological mean}

The GCM we use consists of the hydrodynamical core of MITgcm (Marshall et al. 1997a,b) with idealized thermodynamics. Diabatic heating is parameterized as a Newtonian restoring term (HS94). The model does not include radiative transfer calculations or the water balance. The hydrodynamic core is able to resolve midlatitude baroclinic eddies which play a dominant role in heat transport in the atmosphere. We consider the statistical mean state of the simulated atmosphere. Fig. $8.1 \mathrm{~b}$ shows the temporally and zonally averaged distribution of temperature of the model. 


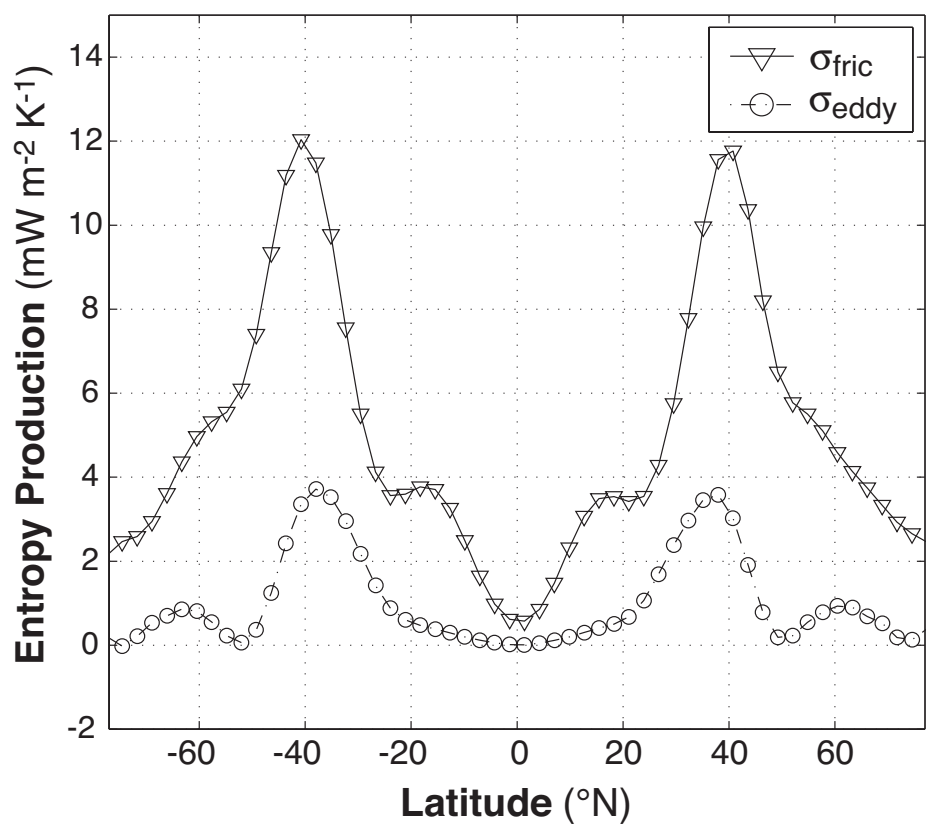

Fig. 8.2. Distribution of zonally averaged entropy production. Triangles and circles represent the frictional and eddy component of entropy production respectively.

Fig. 8.2 shows the distribution of vertically integrated $\sigma_{\text {fric }}$ and $\sigma_{e d d y}$. First, we consider the hemispheric distribution of $\sigma_{\text {fric }}$. In each hemisphere, there is a smaller peak in the tropics and a larger in the mid-latitudes. The dissipation of the mean kinetic energy is responsible for the smaller peak in tropics. The greater peak in the mid-latitudes is due to the damping of the eddy kinetic energy, which dominates global frictional entropy production $\left\langle\sigma_{\text {fric }}\right\rangle$. Observations estimate entropy production by friction to be about $6.5 \mathrm{~mW} \mathrm{~m}^{-2} \mathrm{~K}^{-1}$ (Peixoto et al. 1991; Goody 2000) which compares well with the slightly smaller simulated value of 5.0

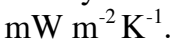

Entropy production by diabatic eddy transfer has a maximum in the midlatitudes. The magnitude of $\sigma_{e d d y}$ is much smaller than $\sigma_{\text {fric }}$, suggesting that diabatic eddy fluxes plays rather minor role in the global entropy production. The magnitude of $\left\langle\sigma_{e d d y}>\right.$ is approximately $1 \mathrm{~mW} \mathrm{~m}^{-2} \mathrm{~K}^{-1}$. Both $\sigma_{e d d y}$ and $\sigma_{\text {fric }}$ have peaks around $35 \mathrm{~N}$ and $35 \mathrm{~S}$, reflecting the significant role of baroclinic eddy transfer in controlling the magnitudes and spatial distribution of entropy production. Combined, the total entropy production in the model is about $6 \mathrm{~mW} \mathrm{~m}^{-2} \mathrm{~K}^{-1}$. 


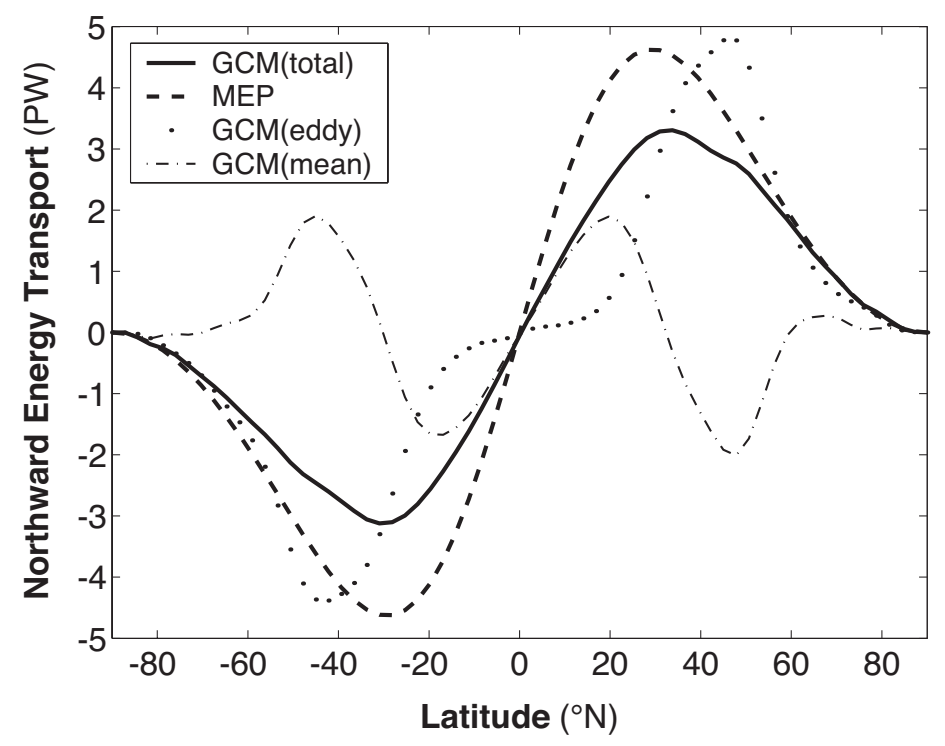

Fig. 8.3. Meridional heat transport of the analytic solution in comparison to the simulated components of the GCM.

\subsubsection{Comparing the analytic MEP solution to the simulated atmosphere}

We test the MEP hypothesis by comparing the simulated temperature distribution and meridional heat transport to those of the analytic MEP solution (Fig. 8.1 and Fig. 8.3). The analytic MEP solution has qualitative similarities to the simulated profile in the mid- and high latitudes. Surface equator-pole temperature difference is in the order of $35 \mathrm{~K}$ in both the MEP solution and the modeled atmosphere. Given the simplicity of the MEP solution in eqn. (8.11), it is rather remarkable that it can capture the gross measure of the large-scale temperature gradient and heat transport.

However, there is a large disagreement in the temperature in the tropics where the simulated potential temperature field shows a uniform distribution. In the upper tropical atmosphere, the Hadley circulation dominates the temperature distribution and energy transport. The disagreement may result from the lack of dynamical constraints in the MEP solution. The MEP solution derived here is based on the energy balance constraint only, but it has been shown that the momentum balance (which is not included) is essential for the dynamics of Hadley cell (Held and Hou 1980).

In Fig. 8.4, we evaluate the MEP solution in terms of the square-root relationship between $T$ and $T_{e q}$. The MEP solution in eqn. (8.11) suggests that the zonally 
averaged absolute temperature is proportional to the square root of the radiative equilibrium temperature profile. To test this scaling relationship, we plot $T$ and $T_{e q}$ in logarithmic scale. The solid line with a slope of 0.5 represents the scaling from the MEP solution. Temperature of low and high altitudes are plotted separately in order to show the qualitative differences between the tropics and high latitudes. The MEP solution compares better with the temperature of high latitudes where $T$ is greater than $260 \mathrm{~K}$. The cold temperatures of the atmosphere in low latitudes tend to have greater slope than 0.5.

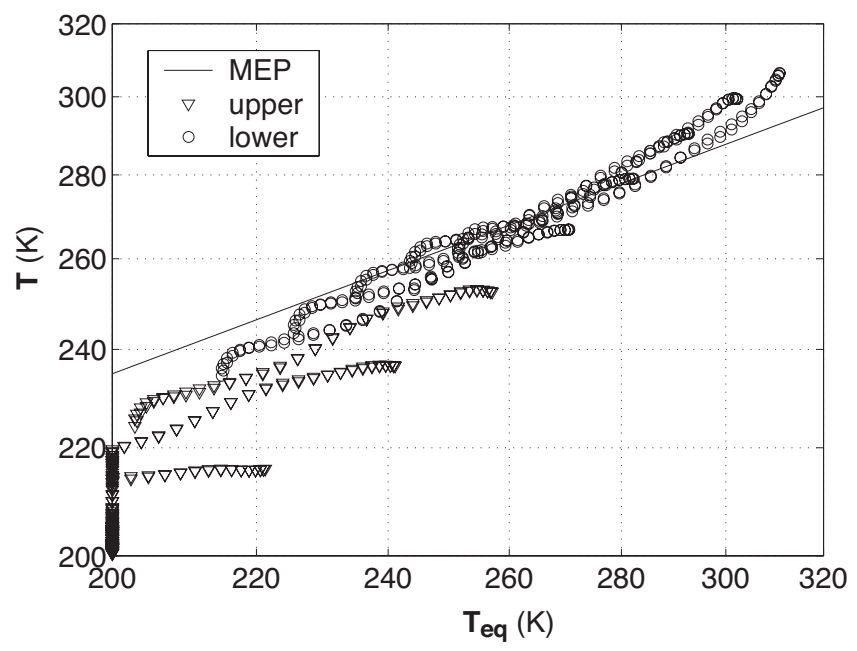

Fig. 8.4. Square root relationship between the $T_{e q}$ and $T$ as expected from MEP. The solid line represents the theoretical MEP solution with a slope of 0.5 . The circles and triangles represent simulated temperatures from the lower $(<500 \mathrm{mb})$ and upper $(>500 \mathrm{mb})$ respectively.

The globally integrated entropy production of the simulated climate $\left\langle\sigma_{T O T}\right\rangle$ is about $71 \%$ of $\left\langle\sigma_{T O T, M E P}\right\rangle$. It is reasonable that the simulated entropy production is somewhat less than the upper bound because of the lack of inclusion of the dynamical constraints. Frictional dissipation is the dominant source of entropy production, contributing approximately $83 \%$ to the total, with the remaining $17 \%$ originating from thermal dissipation. The integral balance of eqn. (8.10) does not exactly hold in the simulation because of spurious source of entropy from numerical diffusion. We find that the entropy production due to numerical dissipation becomes small when the horizontal and vertical resolution of the model is sufficiently high. 


\subsubsection{Sensitivity of entropy production to internal parameters}

In order to understand why the atmospheric circulation would adjust to a state close to MEP, Kleidon et al. (2003) conducted sensitivity simulations with an atmospheric general circulation model similar to the one discussed above (Fraedrich et al. 1998, available for download at http://puma.dkrz.de). Two different methods are used in GCMs to represent turbulent processes such as the development of large-scale eddies in the mid-latitudes and the vertical circulations in the boundary layer at much finer scales.

The dynamics of mid-latitude turbulent mixing is explicitly resolved by the fluid dynamics. However, the spatial resolution of the model is externally prescribed and sets lower limits on the spatial structure of large-scale eddies that can be simulated. Higher model resolutions permit finer structures of the atmospheric circulation, increasing the potential number of modes (or degrees of freedom). Following Dewar (2003, also this volume), we should therefore expect an increase in entropy production with model resolution until sufficiently high degrees of freedom are allowed for by the model resolution. This increase of entropy production up to a certain level is found in the model sensitivity simulations in which the spatial resolution is varied (Fig. 8.5a).
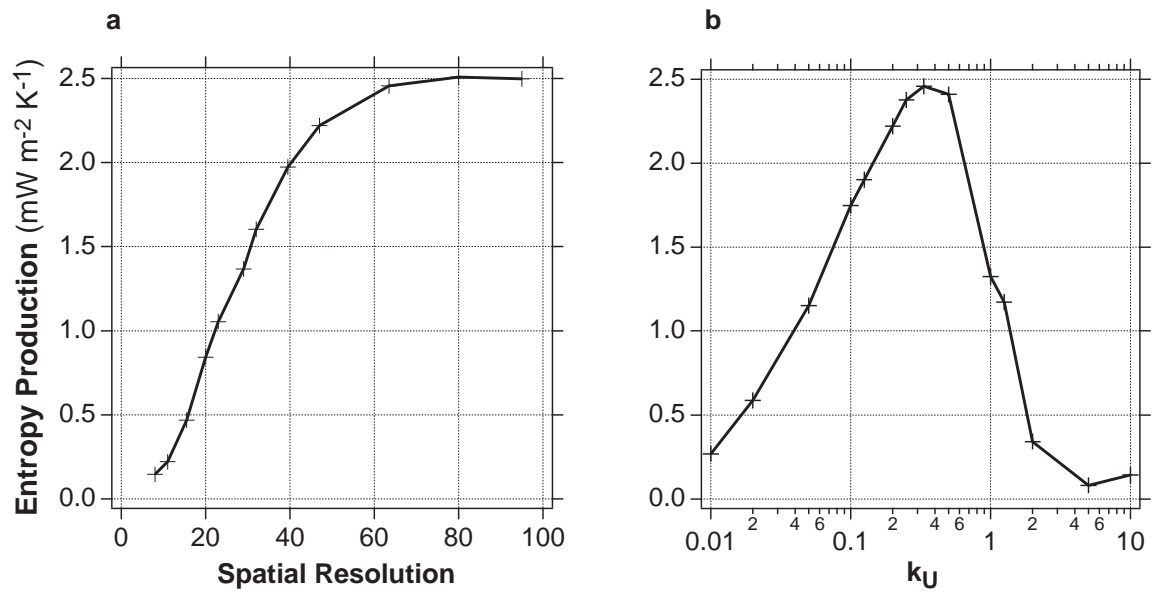

Fig. 8.5. Sensitivity of simulated total entropy production associated with atmospheric heat transport to (a) the model's spatial resolution (expressed by the number of latitudinal bands, with higher values representing finer resolution) and (b) to the frictional coefficient (with higher values representing increased boundary layer turbulence). After Kleidon et al. (2003).

The boundary layer turbulence that develops as a result of surface friction oc= curs at a much finer scale than GCMs are able to resolve. The effect of boundary layer turbulence on the energy and momentum balance is commonly parameter- 
ized in a fairly simple manner. In the idealized GCMs considered here, it is crudely parameterized as a Rayleigh friction term (represented by $k_{U}$ in eqn. 8.3, or described by a friction time scale $\tau_{\text {FRIC }}$ ). For this type of turbulence parameterization, entropy production shows a maximum (Fig. 8.5b), similar to the simple two-box energy balance example which is used to demonstrate the existence of a MEP state (Fig. 1.4). Note that the analytic form of the MEP solution in eqn. (8.11) is not sensitive to this parameter since the maximization does not explicitly include the dynamical constraints of momentum conservation.

The maximum in entropy production in Fig. 8.5 originates from the competing effects of boundary layer turbulence on eddy activity (James and Gray 1986): At the high friction extreme, momentum is rapidly removed, therefore preventing substantial eddy activity. With the reduction in friction intensity, the atmospheric flow becomes increasingly zonal, and therefore more stable to baroclinic disturbances. Consequently, the peak in entropy production corresponds to a maximum in baroclinic activity in the model. (It should be noted in the discussion above that the model does not distinguish between boundary layer turbulence and surface friction. Therefore, the sensitivity to friction should be interpreted as a sensitivity to the characteristics of boundary layer turbulence, and not surface friction per se.) Also note that the rates of entropy production in Kleidon et al. (2003) and shown in Fig. 8.5 are less than the ones obtained above which is likely due to the fact that diabatic heating by friction is not included in their model formulation.

\subsection{Climatological Implications}

In this chapter we have reviewed the thermodynamics of the dry atmospheric circulation, derived a temperature distribution corresponding to a state of MEP, and showed that the simulated temperature fields of an atmospheric general circulation model is broadly similar to the theoretical derived value. Naturally, the considerations used here are subject to some limitations. Most importantly, our focus on the dry atmosphere is limited with respect to the Hadley circulation, since it is driven by the latent heat flux, and therefore explicitly by moist diabatic processes. These processes contribute considerably to the overall entropy production (Pauluis and Held 2002a,b, also Pauluis, this volume). Our theoretical derivation did not include the conservation of potential vorticity, which can explain the fact that the simulated entropy production by the GCM is less than the theoretical estimate. Considering the conservation of potential vorticity is also likely to be important when climates of planets with different rotation rates are considered (which will affect the sensitivity of entropy production to boundary layer turbulence as discussed in the previous section). The important role of the oceanic circulation in contributing to the overall heat transport may also play a role in the distribution of temperature and the state of MEP, but has not been considered here. With these limitations in mind, we nevertheless demonstrated the important role of baroclinic activity for entropy production associated with frictional dissipation in the planetary boundary layer and mixing of warm and cold air masses in the mid-latitudes. 


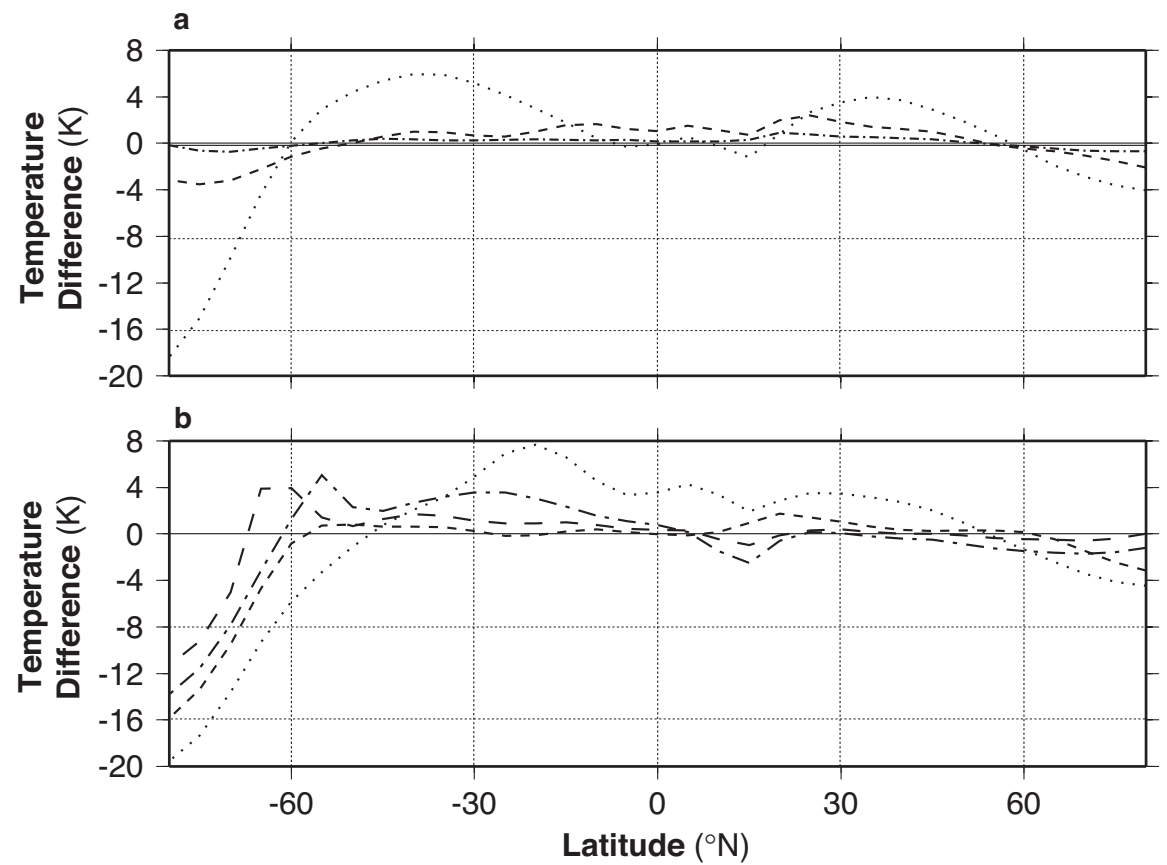

Fig. 8.6. Difference in the latitudinal variation of temperatures for the lowest atmospheric model layer in comparison to the simulated climate of maximum entropy production (for a southern hemisphere winter setup). (a) effects of different model resolutions between $\mathrm{T} 10$ and $\mathrm{T} 42$ resolution (dotted), same for T21 (dashed), and T31 (dash-dotted) resolution, each with optimum values of $\tau_{F R I C}$. (b) effects of different intensities of boundary layer turbulence between $\tau_{F R I C}=0.1$ day and $\tau_{\text {FRIC }}=3$ days (dotted), same for $\tau_{F R I C}=1$ day (short dashes), same for $\tau_{\text {FRIC }}=10$ days (long dashes), same for $\tau_{F R I C}=100$ days (dash-dotted), each at T42 resolution. After Kleidon et al (2003).

Furthermore, we have shown that the state of MEP as simulated by the simple GCMs used here is sensitive to the model parameterization of boundary layer turbulence and model resolution. If we take the state of MEP as representative of the macroscopic steady-state atmospheric circulation, then these sensitivities can have important implications for the application of GCMs to climate research. Since MEP represents the state of highest baroclinic activity, it also is associated with the most effective heat transport to the poles. This leads to the least temperature gradient $\Delta T_{E, P}$ for the simulation with MEP in comparison to simulations with lower model resolution or other intensities of boundary layer friction (Fig. 8.6). These model results suggest that in comparison to MEP, any other macroscopic state of the atmospheric circulation would show less baroclinic activity, and therefore transport less heat to the poles, leading to an overestimation of the equatorpole temperature gradient. This in turn may have important consequences for the 
adequate simulation of climatic change. It is generally known that GCMs tend to overestimate $\Delta T_{E, P}$ in paleoclimatology, for instance during periods of high carbon dioxide concentrations of the Eocene (Pierrehumbert, 2002). Following the line of reasoning presented here, this may simply be an artifact of a GCM setup which does not represent a MEP climate.

Acknowledgements. T. I. would like to thank John Marshall for introducing the author to the MEP hypothesis, fruitful discussion and helpful comments. A. K. conducted this research in collaboration with Klaus Fraedrich and his research group and thanks for stimulating discussions and hospitality during the author's stay in July 2003. A. K. gratefully acknowledges financial support from the National Science Foundation though grant ATM0336555 .

\section{References}

Dewar R.C., (2003) Information theory explanation of the fluctuation theorem, maximum entropy production, and self-organized criticality in non-equilibrium stationary states. $\mathbf{J}$ Physics A 36: 631-641.

Goody R., (2000) Sources and sinks of climate entropy, Q J R Meteorol Soc, 126(566), 1953-1970.

Grassl H., (1981) The climate at maximum-entropy production by meridional atmospheric and oceanic heat fluxes, Q J R Meteorol Soc, 107 (451), 153-166.

Held I.M., A.Y. Hou, (1980) Non-linear axially symmetric circulations in a nearly inviscid atmosphere, J Atmos Sci, 37 (3), 515-533.

Held I.M., M.J. Suarez, (1994) A proposal for the intercomparison of the dynamical cores of atmospheric general circulation models., Bull Am Met Soc, 75(10), 1825-1830.

James IN, Gray LJ (1986) Concerning the effect of surface drag on the circulation of a planetary atmosphere, Q J R Meteorol Soc, 112, 1231-1250.

Kleidon A, Fraedrich K, Kunz T, Lunkeit F (2003) The atmospheric circulation and states of maximum entropy production, Geophys Res Lett 30(23), 2223.

Lorenz E.N., (1960) Generation of available potential energy and the intensity of the general circulation, in Dynamics of Climate, edited by R.L. Pfeffer, 86-92, Pergamon Press, Oxford.

Lorenz R.D., (2001) Titan, Mars and Earth: Entropy production by latitudinal heat transport, Geophys Res Lett 28 (16), 3169-3169.

Marshall et al, (1997a) Hydrostatic, quasi-hydrostatic, and non-hydrostatic ocean modeling, J Geophys Res 102(C3), 5733-5752.

Marshall et al, (1997b) A finite-volume, incompressible Navier Stokes model for studies of the ocean on parallel computers, J Geophys Res 102(C3), 5753-5766.

Nicolis G., C. Nicolis, (1980) On the entropy balance of the earth-atmosphere system, Q J R Meteorol Soc, 106 (450), 691-706.

Ozawa, H., A. Ohmura, (1997) Thermodynamics of a global-mean state of the atmosphere - A state of maximum entropy increase, J Clim, 10, 441-445.

Ozawa, H., S. Shimokawa, and H. Sakuma, (2001) Thermodynamics of fluid turbulence: A unified approach to the maximum transport properties, Phys Rev E, 64, 026303. 
Ozawa H, Ohmura A, Lorenz RD, Pujol T (2003) The second law of thermodynamics and the global climate system - A review of the maximum entropy production principle, Rev Geophys 41: 1018.

Paltridge G.W., (1975) Global dynamics and climate --- System of minimum entropy exchange, Q J R Meteorol Soc, 101, (429), 475-484.

Paltridge G.W., (1978) Steady-state format of global climate, Q J R Meteorol Soc, 104 (442), 927-945.

Pauluis O., I.M. Held, (2002a) Entropy budget of an atmosphere in radiative-convective equilibrium. Part I: Maximum work and frictional dissipation, J Atmos Sci, 59 (2), 126-139.

Pauluis O., I.M. Held, (2002b) Entropy budget of an atmosphere in radiative-convective equilibrium. Part II: Latent heat transport and moist processes, J Atmos Sci, 59 (2), 140-149.

Peixoto J., A. Oort, M. Almeida, and A. Tome, (1991) Entropy budget of the atmosphere, J Geophys Res, 96 (D6), 10981-10988.

Pierrehumbert R.T. (2002) The hydrologic cycle in deep-time climate problems, Nature, 419 (6903), 191-198.

Pujol T., J.E. Llebot, (2000) Extremal climatic states simulated by a 2-dimensional model Part I: Sensitivity of the model and present state, Tellus, 52A (4), 422-439.

Shimokawa S., H. Ozawa, (2002) On the thermodynamics of the oceanic general circulation: Irreversible transition to a state with higher rate of entropy production. Q J R Meteorol Soc, 128 (584), 2115-2128.

Shutts G. J., (1981) Maximum entropy production states in quasi-geostrophic dynamical models, Q J R Meteorol Soc, 107 (453), 503-520. 
